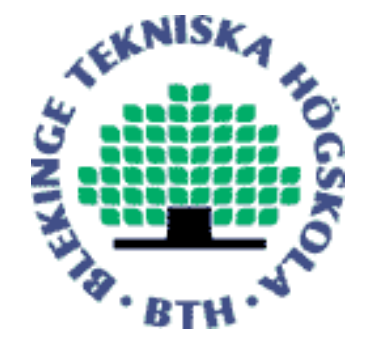

Copyright (C) 2012 IEEE.

Citation for the published paper:

Influence Map-based Controllers for Ms. PacMan and the Ghosts

Johan Svensson, Stefan Johansson

IEEE Conference on Computational Intelligence and Games

2012 Granada

This material is posted here with permission of the IEEE. Such permission of the IEEE does not in any way imply IEEE endorsement of any of BTH's products or services Internal or personal use of this material is permitted. However, permission to reprint/republish this material for advertising or promotional purposes or for creating new collective works for resale or redistribution must be obtained from the IEEE by sending a blank email message to pubs-permissions@ieee.org.

By choosing to view this document, you agree to all provisions of the copyright laws protecting it. 


\title{
Influence Map-based Controllers for Ms. PacMan and the Ghosts
}

\author{
Johan Svensson Student member, IEEE and Stefan J. Johansson, Member, IEEE
}

\begin{abstract}
Ms. Pac-Man, one of the classic arcade games has recently gained attention in the field of game AI through the yearly competitions of various kinds held at e.g. CIG. We have implemented an Influence Map-based controller for Ms. Pac-Man as well as for the ghosts within the game. We show that it is able to handle a number of various situations through the interesting behaviors emerging through the interplay of the different maps. It is also significantly better than the previous implementations based on similar techniques, such as potential fields.
\end{abstract}

\section{INTRODUCTION}

Since 2007, there have been yearly competitions in implementing Ms. Pac-Man controllers [1]. The competitions have lately also addressed the problem of controlling the ghosts [2].

We intend to investigate if an influence map-based solution would be applicable to control Ms. Pac-Man, in particular if it would be possible to achieve better results than previous attempts using similar approaches. First, we will give the readers some background about Ms. Pac-Man, the previous work in the area, and an overview of potential fields and influence maps in the context of Ms. Pac-Man. Then we go through the implementation of an influence map-based Ms. Pac-Man controller, as well as its Ghost counterpart. We then describe the setup of the validations of these implementations, as well as the results they gained. In the end, we finish with a discussion, some conclusions and line out some future directions of research.

\section{A. Ms. Pac-Man}

Ms. Pac-Man (Midway, 1981) is the successor of the original Pac-Man, released by Namco in 1980. The player controls a female yellow character (Ms. Pac-Man) that eats pills in a maze. In the implementation that we have used for our experiments (the CIG 2012 version of the simulator) our ghosts chase her according to the following simple rules (a.k.a. the starter ghosts):

- A ghost is never allowed to choose to go in the direction it just came from.

- If a ghost moves along a corridor, it has no other choice than to continue moving. It can only make decisions on how to move when it enters a crossing. If so:

- In case Ms. Pac-Man is either in power mode (she has just eaten a power pill), or is closer to a power pill than 15 positions, the ghosts choose the paths that take them as far away as possible from the current position of Ms. Pac-Man.

Johan Svensson and Stefan J. Johansson are both with Dept. of Computing at Blekinge Institute of Technology
- Otherwise, the ghosts choose to go in the directions of the shortest paths to the current position of Ms. Pac-Man in $90 \%$ of the cases.

- In the rest $10 \%$ of the cases, they choose a random legal direction to move in (including the shortest path direction described above).

The counter-measure of the player is the possibility to eat power pills that turn the ghosts into slow-moving edible rewards for a limited amount of time. There are four power pills at each level, one near each corner of the map. Once a ghost is eaten, it spawns in the center area of the screen as an ordinary ghost. In power mode, the first ghost is worth 200, the second 400, the third 800 and the fourth 1600 points.

When Ms. Pac-Man has eaten all of the pills at a level, she is teleported to a new level with new pills. The same happens if the time spent at a level exceeds 3000 game tics. The player has in total three lives to spend.

\section{B. Previous work}

Early attempts to construct Pac-Man controllers include the work of Koza [3] and Gallagher and Ryan [4]. Since PacMan, in contrast to Ms. Pac-Man, uses deterministic rules for the movements of the ghosts, Koza's genetic programmingbased approach learnt to exploit the static ghost movements in the game (a game that he implemented himself; for further analysis of the strengths of this approach, see [5]). Gallagher and Ryan used a weighted set of rules that evolved over time based on the results of the previous game.

Since 2008, there is a yearly competition held at the Computational Intelligence and Games (CIG) conference. This initiative has also lead to an increased level of research activities in the area of Ms. Pac-Man controllers with a number of different solutions presented.

Wirth and Gallagher proposed a solution in 2008 [6] claimed to be based on influence maps. Despite the title, they used potential fields, rather than influence maps to control Ms PacMan. We will discuss this solution more in detail in Section I-C.

Bell et al. used a tree search based solution that utilized the information about the direction of the ghosts revealed by their gaze. They also used pre-calculated paths to find the way to the closest pills [7].

DeLooze and Viner proposed a fuzzy Q-learning approach that was able to adapt its strategy to new situations in the game [8].

Martin et al. proposed an artificial ant-based solution and used genetic algorithms to optimize the parameters of the ants [9]. 
Robles and Lucas used a tree-based search reaching a depth of 40 moves ahead in the search, which showed to be deep enough to get very good scores [10].

Their work was further developed by Samothrakis et al. that used Monte Carlo tree search with limited tree depth to find even better paths [11].

\section{Potential Fields vs. Influence Maps}

Potential fields and Influence maps are techniques that address similar kinds of problems using similar ideas. They both have the notion of attractive and repelling sources.

In the case of potential fields, a goal may attract its surroundings by emitting a field with a strength that is a function of e.g. the Euclidean distance between the source and the probing points, thus making a unit that has to choose between a number of lookahead positions to go to, to choose the position with the highest probed potential.

In the case of influence maps, the goal would influence its adjacent tiles, that in turn influence its adjacent tiles, etc. in a manner that propagates the influence of the source arbitrarily far away. The same unit moving towards the same goal in such a setting would then in the same way compare the influences in its lookahead positions, choosing the one with the most attractive influence (since that one is closest to the goal if the influence is decreasing with the distance).

Both techniques may of course use several sources, and the sources may be either attractive, or repelling.

There is an important distinction between these two perspectives; whereas potential field calculations are fast (just calculate the distances to each and on of the objects of interest from the probing position), they are unable to take the terrain of the map into account, i.e. it can not recognize that there is a wall between the pill and the probed position, see Fig. 1. As can be seen in Fig. 2, influence maps do not struggle with that problem. Their propagation of influences can be terrain sensitive, meaning that we can stop the propagations where a wall would stop us from moving. By doing so, the propagations instead follow the paths in the maze. In the case of Ms. Pac-Man, there is one important simplification of the calculations that can be done; since the influences are not propagated at all through the walls, but are solely following the paths in the maze, we may use the shortest path to calculate the influence. ${ }^{1}$

Despite this optimization, it is still computationally a bit more expensive, since the calculations of shortest paths in most cases takes more time than to calculate Euclidean distances.

\section{Description OF THE MS PACMAn CONTROLler}

\section{A. The Lookahead Positions}

The controller can move Ms. Pac-Man in four different directions, Left, Right, Up, and Down. We use the directions

\footnotetext{
${ }^{1}$ In the general case, the influences have to be propagated in an iterative way. This also allows for complex interaction with the underlying terrain, e.g. a dense forrest may slow units down, or a river may speed up or slow down a boat depending on the direction. From an influence map point of view, this corresponds to various conductivities in the calculation of the propagation of influences.
}

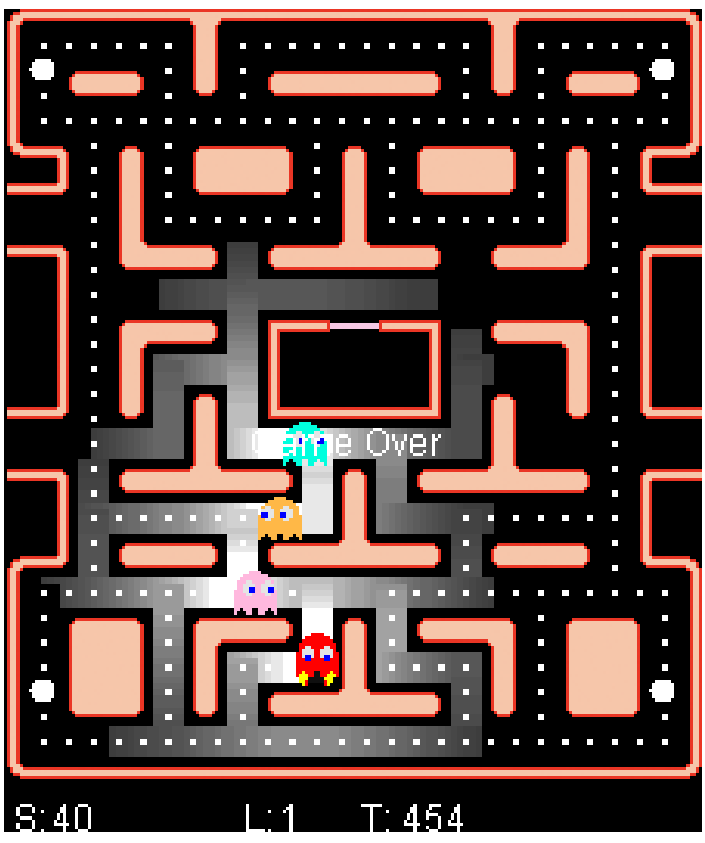

Fig. 1. The aggregated potential field used by Ms. Pac-Man. Light areas are repelling. Note how the repelling field spreads through the walls and in all directions around the ghosts.

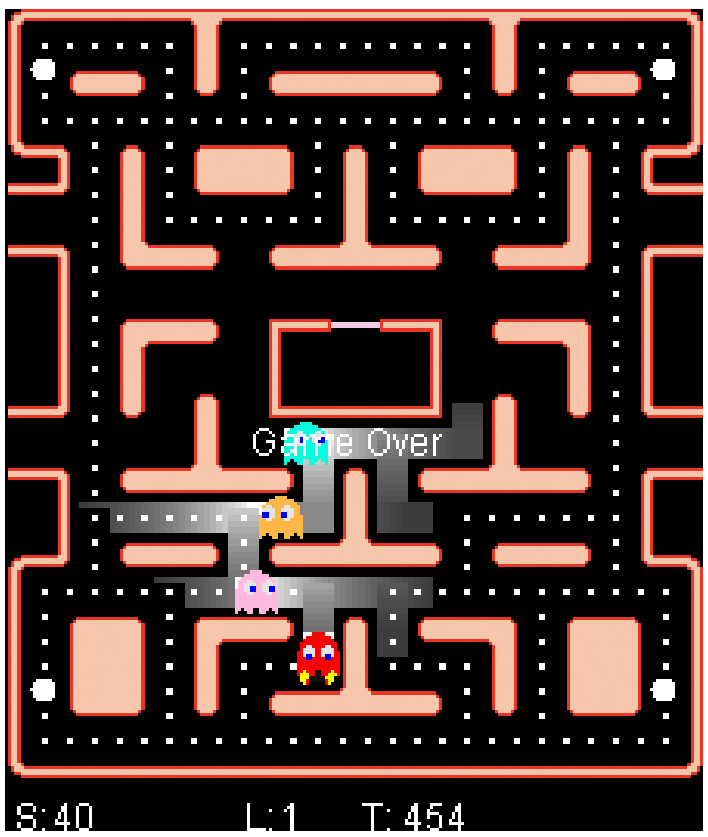

Fig. 2. The aggregated influence map used by Ms. Pac-Man. Light areas are repelling. Here we see an example of how the repelling influences propagate through the maze. Note that they do not propagate in the directions in which the ghosts cannot move (the directions they came from). 
that allow valid movements to decide what lookahead positions to use. In a corridor, that means two directions, whereas in a junction we have three or four lookahead positions to evaluate.

\section{B. The Influences of Pills}

The Influences of Pills are designed to make Ms. Pac-Man be attracted by the positions of the pills that are left to eat at the current level in the game. Each pill emits a value of 4, which is multiplied by 0.95 for each position the lookahead position, or lap is further away from the pill:

$$
I(l a p, p i l l)=4 \cdot 0.95^{d_{A *}(l a p, p i l l)}
$$

where $d_{A *}($ lap, pill $)$ is the distance measured by $A *$ between the lap and the pill.

The power pills are not included in this map; they are handled separately.

The total influence from the pills is calculated as follows:

$$
I_{\text {IoP }}(l a p)=\sum_{p \in P} I(l a p, p)
$$

where $P \subset S$ is the set of pills left to eat at a level.

\section{The Influence of Ghosts}

The Influence of Ghosts is the most important influence map in the simulation. Avoiding the ghosts is the most vital part for Ms. Pac-Man since the ghosts are the only objects that can end the game. The influence maps that the ghosts emit are negative repelling maps that Ms. Pac-Man wants to move away from. The exceptions are the directions from where the ghosts just came which are considered to be safe. Each ghost $g$ emits a negative value $I o G$, with a multiplier of 0.95 , i.e.

$$
I_{I o G}(l a p, g)=I o G \cdot 0.95^{d_{A *}(l a p, g)}
$$

The ghosts have a limited distance that they influence. The distance that they influence is set to $\leq I o G / 10$. If Ms. PacMan is within the influence distance of several ghosts, is only the most negative value that influences Ms. Pac-Man:

$$
I_{I o G}(l a p)=\min _{g \in G} \cdot I_{I o G}(l a p, g)
$$

\section{The Influence of Edible Ghosts}

The Influence of Edible Ghosts is activated during the period right after Ms. Pac-Man has eaten a power pill. The duration of that period varies, and gets shorter and shorter as the level of the game increases. It is propagated in the same way as the IoG, but of course with an attractive influence, and at all sides of the ghosts (not only in the direction of travel as for the IoG), i.e.

$$
I_{I o E G}(l a p, g)=I o E G \cdot 0.95^{d_{A *}(l a p, g)}
$$

where $I o E G$ is the positive weight of the edible ghost. The values of several edible ghosts are combined in the same way as for the ordinary ghosts:

$$
I_{\text {IoEG }}(\text { lap })=\max _{g \in G} \cdot I_{I o E G}(\text { lap }, g)
$$

\section{E. The Influence of Power Pills}

The Power Pills emit positive influences when the combined value of all four ghosts distances is of less than $d$ and emit negative influences when the ghosts are further away. This is only calculated for the closest Power Pill PP:

$$
\begin{aligned}
& I_{I o P P}(l a p)= \\
& \text { IoPP. } \begin{cases}-\left(0.95^{d_{A *}(\text { lap }, P P)}\right) & \sum_{g \in G} d_{A *}(g, P P)<d \\
0.95^{d_{A *}(l a p, P P)} & \sum_{g \in G} d_{A *}(g, P P) \geq d\end{cases}
\end{aligned}
$$

This means that Ms. Pac-Man does not eat the Power Pills when the ghosts are out of reach. The scores for eating the ghosts are 200, 400, 800 and 1600 respectively for the first, second, third, and fourth ghost eaten during the power period. If a new power pill is eaten before the power period of a previous power pill ends, the ghost counter is reset and the next ghost eaten will only give the player 200 points.

\section{F. The Influence of Freedom of Choice}

When Ms. Pac-Man is being cornered by two or more ghosts, she wants to find the nearest junction to escape the ghosts. Ms. Pac-Man measures the values from the 2 adjacent nodes and if the sum of these nodes negatively influences is below a certain threshold, the nearest junction starts emit a positive influence. In other words, if Ms. Pac-Man senses negative influences from ghosts in both directions in a corridor, she will be highly attracted by the nearest junction where she may have a chance to escape.

$$
\begin{aligned}
& I_{\text {IoFoC }}(\text { lap })= \\
& \begin{cases}\text { IoFoC } \cdot 0.95^{d_{A *}(\text { lap }, J)}, & I_{\text {IoG }}(\text { lap })+I_{\text {IoG }}(\text { lbp }) \\
0, & <\text { IoFoC } \\
0 & \text { otherwise }\end{cases}
\end{aligned}
$$

where $J$ is the position of the nearest junction and $l b p$ is the look behind position, the position just visited by Ms. Pac-Man.

\section{G. The Combination of the Influences}

The overall influence $I$ (lap) of a lookahead position lap is calculated as:

$$
I(l a p)=\sum_{m \in I M} I_{m}(l a p)
$$

where $I M$ is the set of all influences in our solution, i.e. $I M=\{I o P, I o G, I o E G, I o P P, I o F o C\}$

\section{The Ghost Controller}

The ghost controller is implemented in a similar fashion as the Ms. Pac-Man controller. We use two major influences:

- An Influence of Ms. Pac-Man is in general attractive for the ghosts.

- The Influence of Ghosts is a repelling field that gives the ghosts an incentive to keep a certain distance to each other. 
In both cases, the position of the nearest power pill in relation to Ms. Pac-Man is of importance. The closer she is to the power pill, the more dangerous it is for a ghost to follow her and the more dangerous it is for the ghosts to travel right next to each other, since it will improve Ms. Pac-Man's chances to eat them all within the power period.

\section{A. The Lookahead Positions of the Ghosts}

The ghosts do not have the same freedom as Ms. Pac-Man to choose where to go. They are bound by the following rules:

- In the corridors, the ghosts can only move in the direction from which they entered, i.e. a ghost cannot change direction in the middle of a corridor.

- In an intersection, a ghost can choose any valid direction. Valid directions are all open directions except the one the from which the ghost entered the intersection.

Since the ghosts may only make one move unless they are in an intersection, the influence map based decision making is only activated when needed.

\section{B. The Influence of Ms. Pac-Man}

Ms. Pac-Man's field can both be negative and positive depending on what distance she has to the closest Power Pill. When Ms. Pac-Man comes close to a Power Pill she starts to send out negative influence to keep the ghosts away instead of attract them. If she eats the Power Pill the possibility of eating all of the ghosts is then less likely. During the effect of the Power Pill, Ms. Pac-Man sends out only negative influences, making the ghosts trying to avoid her. The influence of Ms. Pac-Man is based on the following equations:

$$
\begin{gathered}
\alpha=\min _{p \in P P} d_{A *}(M P M, p) / c_{P P} \\
\text { IoMPM }= \begin{cases}200 \cdot \alpha^{d_{A *}(\text { lap }, M P M)}, & \alpha>0.85 \\
-200 \cdot(1.5-\alpha)^{d_{A *}(\text { lap }, M P M)}, & \alpha \leq 0.85\end{cases}
\end{gathered}
$$

where $c_{P P}$ is a fixed value that determines the distance when Ms. Pac-Man switches from sending out positive influence to instead send out negative influences in order to keep the ghosts away if a Power Pill is eaten. The value of $c_{P P}$ is varied in the experiments.

\section{The Influence of Ghosts}

Using the influence map we can send out negative influences around the ghost that repel other ghosts and prevent them of coming close to each other. By doing so they should avoid taking identical paths to Ms. Pac-Man and thus increase the chance of trapping her. Also they should be more spread when Ms. Pac-Man eats a power pill. When the ghosts are far away from each other they have a larger influence range and when close to Ms. Pac-Man the negative influence range is shorter. The reason for closing in the range is to avoid a situation where the ghosts are repelling each other from different sides of Ms. Pac-Man and thus none of them go for her. The formula used is:

$$
I(\text { lap })=\gamma \cdot 0.90^{d_{A *}(\text { lap }, \text { ghost })},
$$

where $\gamma$ is the weight of the ghost and $d_{A *}$ (lap, ghost) is the distance calculated by $\mathrm{A}^{*}$ between the lookahead position and the other ghost. To determine the range that the ghost influences we use:

$$
R=\gamma-d_{A *}(\operatorname{lap}, M P M) \cdot \beta / d_{A *}(\text { ghost, } M P M)
$$

If $R$ is positive we continue to increase the range of the influence. $\gamma$ is the weight of the ghost, and $\beta$ is a constant value corresponding to the distance between Ms. Pac-Man and the Power Pill at which Ms. Pac-Man changes from being attractive to being repelling. A higher value means that a longer distance when the range start to decrease and a smaller value means a shorter distance to Ms. Pac-Man before the negative influence start to shorten.

\section{EXPERIMENTAL SETUP}

The experiments were done by implementing a Ms. PacMan and a Ghost controller that followed the rules of the CIG2012 Ms. Pac-Man vs. Ghost competition.

\section{A. The Ms. Pac-Man controller experiments}

At first there were seven parameters that could be tested, but seven parameters and 100 iterations for each parameter would mean $100^{7}$ test cases and 40 trial runs for every case would take years to complete. The tests are now done with five iterations for each parameter except the last that is eight since the experiment is done on a PC with eight cores to maximize the efficiency. The five parameters that are now used are the positive value for pills, power pills, edible ghosts, the negative value for the ghosts, and the threshold value when Ms. PacMan wants to find the nearest junction, see Table I. Now there are only 5000 test cases with ten trial runs each, resulting in a 14 hour long experiment.

The Ms. Pac-Man controller is tested against the starter ghost controller that came with the game. It is a primitive, quite aggressive controller as we described in the introduction.

TABLE I

THE PARAMETER SPACE OF OUR EXPERIMENT.

\begin{tabular}{|l|l|}
\hline Parameter & Values \\
\hline Power pill influence (IoPP) & $0,10,20,30,40$ \\
Pill influence (IoP) & $0,2,4,6,8$ \\
Edible ghost influence (IoEG) & $0,30,60,90,120$ \\
Ghost influence (IoG) & $0,-30,-60,-90,-120$ \\
Freedom of choice infl. (IoFoC) & $0,30,60,90,120,150,180,210$ \\
\hline
\end{tabular}

\section{B. The Ghost controller experiments}

The Ghost controller uses three different parameters. The value for each ghost, $\gamma$, a Power Pill distance factor, $c_{P P}$, and a distance factor to Ms. Pac-Man, $\beta$ resulting in 1000 experiment configurations, each run ten times. The Ms. PacMan controller to be used against the Ghost controller was a controller that came with the game. However, there is a big difference here since the Ms. Pac-Man controller needs more logic to be effective than what the Ghost controller needs. In the Ghost controller experiment, we have tried to 
find good values of the parameters presented in Table II. Just as in the case of the Ms. Pac-Man controller, we have run one experiment per combination of parameter values.

TABLE II

THE PARAMETER SPACE OF OUR EXPERIMENT.

\begin{tabular}{|l|l|}
\hline Parameter & Values \\
\hline Ghost weight $\gamma$ & $0,-30,-60,-90,-120,-150,-180,-210$, \\
& $-240,-270$ \\
Power pill distance factor & $0,6,12,18,24,30,36,42,48,54$ \\
$c_{P P}$ & \\
Ms. Pac-Man Influence $\beta$ & $0,20,40,60,80,100,120,140,160,180$ \\
\hline
\end{tabular}

\section{EXPERIMENTAL RESULTS}

We have run a long series of experiments and we present it in a narrowing style, starting by eliminating the effect of the most significant parameters by studying how the landscape looks for pairs of parameters, given the average results of all possible combination of values of the other parameters. Since also the worst values of the other parameters are part of the average score calculation, the average score is not very high. However, we can sort out which parameters that have the greatest impact on the result, since that will show the greatest difference between its maximum and minimum value. We lock these two most significant parameters at their optimal values and redo the analysis with the rest of the unbound parameters.

\section{A. The Ms. Pac-Man Controller Experiments}

We have run one game with each setup, i.e. $5^{4} \cdot 8=5000$ games. The first part of the analysis deals with identifying the most significant pair of parameter values. This is done by looking at the average scores of each combination of parameter values, when the other parameters are varied.

The other parameters generate between 125 and 200 combinations, meaning that there is a rather wide variety of setups to base the result on. The results are presented in Figs. 3-7. We see that the highest scores are achieved through combining IoFoC (90) with IoG (-90) peaking at an average score of 7913.

The next step of the analysis of the experiments is to lock the dominating fields at their optimal setting. When we set both IoFoC to 90 and FoG to -90 , and continue to vary the other three, we get the results illustrated in Fig. 8. It is clear that the third most significant parameter is the value of the edible ghost, where a value of 60 seems to yield the best scores.

The last step is to look at the results when the three most significant parameters are locked to their (averaged) best positions: $\mathrm{IoFoC}=90$, IoEG $=60$ and $\mathrm{IoG}=-90$. The result is shown in Fig. 9 with a peak at IoPP $=40$ and IoP $=4$ (score $=15.140)$.

\section{B. The Ghost Controller Experiments}

The results from the experiments show that the highest score is achieved through the values $\gamma=-20$ and $\beta=0$. These two variables are used together and with $c_{P P}=20$, the range

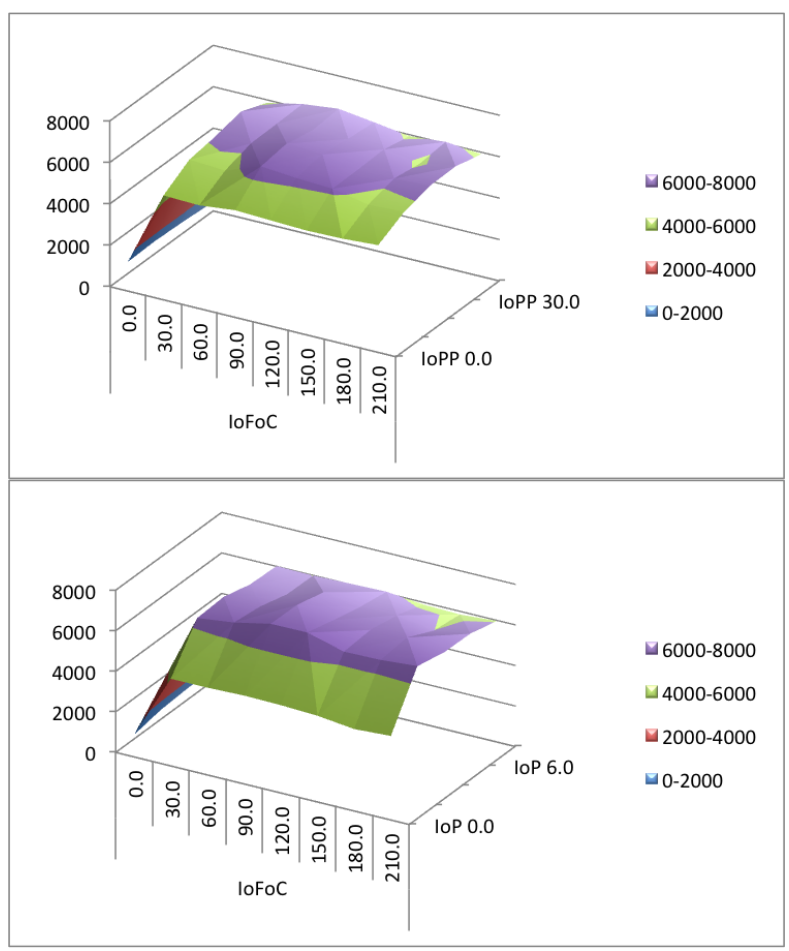

Fig. 3. The score of Ms. Pac-Man given the value of the Influence of Freedom of Choice, and the Influences of Power Pill (top) and Pill (bottom) respectively.

of the negative influence is practically 0 . It only emitted in its closest vicinity peaking at $c_{P P}=12$, this means that Ms. Pac-Man can walk very close to the Power Pill before she starts to send out negative influences.

The results of the Ghost controller experiments are presented in Fig. 10.

\section{DISCUSSION}

\section{A. The Ms. Pac-Man controller}

The Ms. Pac-Man controller was easy to implement due to the very good conditions of the game and the node system that the game uses. Our first concern about the use of influence maps was the memory usage. Due to a large amount of nodes that holds data about the positive and negative influences we though that we might have to lower the precision of the nodes. However, the memory usage was far from the limit of the game that is $512 \mathrm{MB}$.

Ms. Pac-Man suffers from the ability to only see one node ahead of itself and dies a lot due to the fact that when a group of pills are in a corner with only one exit as Ms. Pac-Man eats those pills she gets trapped between two ghosts. The fix to address this problem is to find the nearest junction and it works well in the middle parts of the maps where the corridors are short and there a lot of junctions. However, at the edges of the map where longer corridors and few junctions are present our implementation does not work very well.

In early testing of the ghost we used a quite strong repelling value for the ghosts, -300 to be exact. The distance that the 


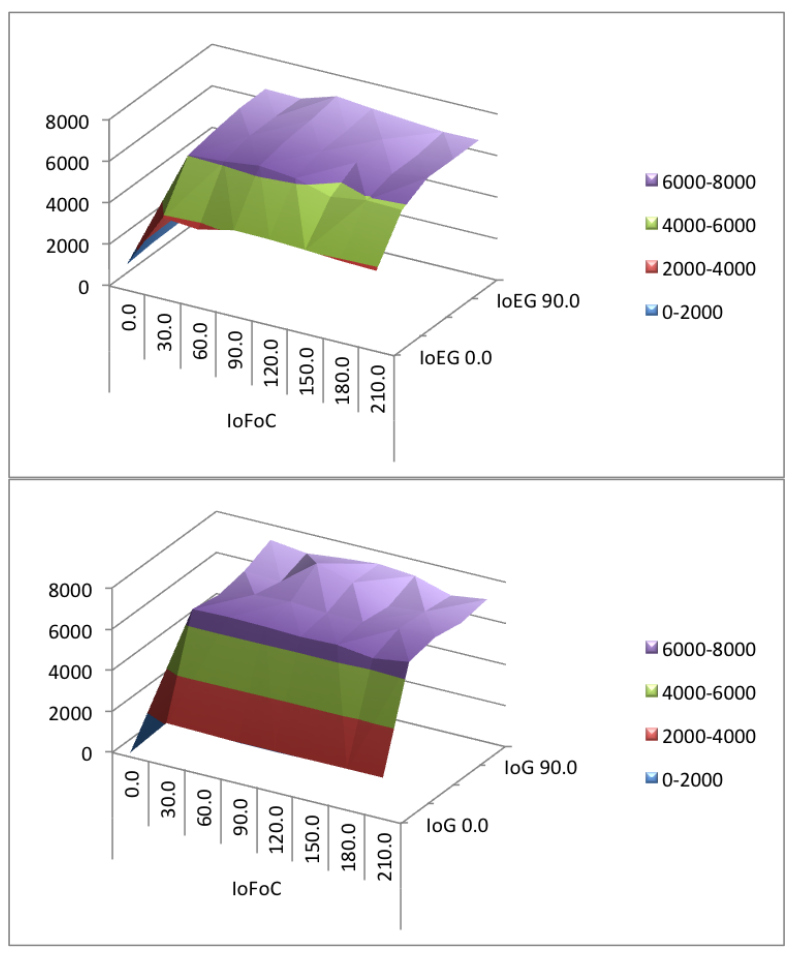

Fig. 4. The score of Ms. Pac-Man given the value of the Influence of Freedom of Choice, and the Influences of edible Ghosts (top) and Ghosts (bottom) respectively.

influence is propagated is depending on the value of the ghost. With a higher value this meant that Ms. Pac-Man got pushed around quite a lot and often became cornered in the end with no exit. The test results showed that with a much lower value of the ghosts they have a shorter influence range and this meant that Ms. Pac-Man had more space to move around before needing to escape from the ghosts.

\section{B. The ghost controller}

When testing with bigger ranges of repelling influences from the ghosts, the repelling influence got too strong so when two ghosts were far away from Ms. Pac-Man, the repelling influence overpowered the attracting influence of Ms. PacMan. This resulted in ghosts walking around in circles.

The best test result came when the range and power from the ghosts negative influence is very short and weak. This resulting in that the ghosts always know where Ms. Pac-Man is and do not care at all about the other ghosts.

\section{Discussion of the method}

The way in which we identify promising areas of values in the parameter space could be improved, e.g. by using a genetic algorithm optimization. Influence maps as well as potential fields are both quite robust methods, not very sensitive to the strength of the influence; often other types of parameters have a greater impact. This does not mean that it cannot be improved. By using a GA-based optimization, we may better explore promising areas to find even better configurations.

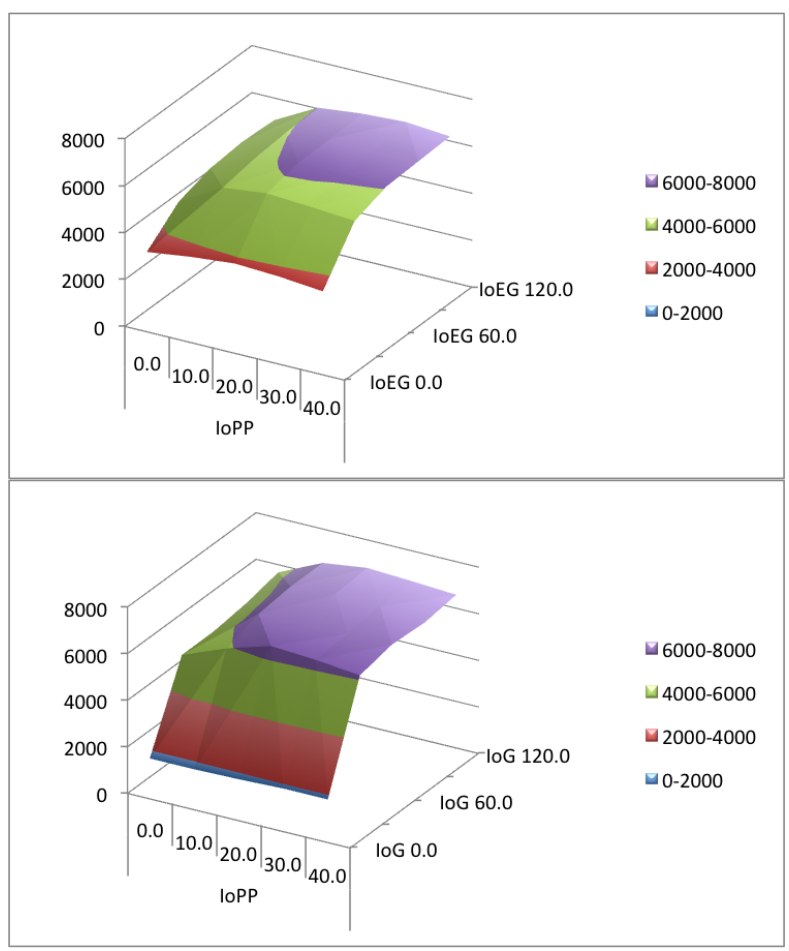

Fig. 5. The score of Ms. Pac-Man given the value of the Influence of Power Pills, and the Influences of edible Ghosts (top) and Ghosts (bottom) respectively.

We used "only" 10 games per combination of parameters as a basis for the results. This does not seem to be a problem in the first diagrams, where the landscapes of scores seems to be rather stable and smooth, but then bear in mind that the results in e.g. Fig. 5 are based on the average of $5 \cdot 5 \cdot 8$ parameter settings, each run 10 times, i.e. 2000 scores. As we lock the values of the parameters, that number quickly decreases to be no more than 10 runs for each position in Fig. 9.

The scores also show a quite high variation, the highest score that the controller achieved was 34170 , so a higher number of runs would probably have to be done to get stable results also at the level of each parameter combination.

\section{Related studies}

Wirth and Gallagher made a similar implementation, but based the distance calculations on Euclidean distances rather than the shortest distances in the maze [6]. This detail may sound extraneous, but the authors identify the use of Euclidean distance measures as one of the weaknesses in the study and bring up the possibility to use a more accurate measure as a future work option. Score-wise, we have shown that it is possible to get scores that averages at 15.775 points against standard ghosts (the average of 10 games at that setup).

In relation to the top scoring controllers in the world, our solution is by far not at their level of performance. However, there is an important difference in what kind of solutions we use. Our solution is purely reactive. It looks at the current state of the game, at the action space(s) of the unit(s) and act 


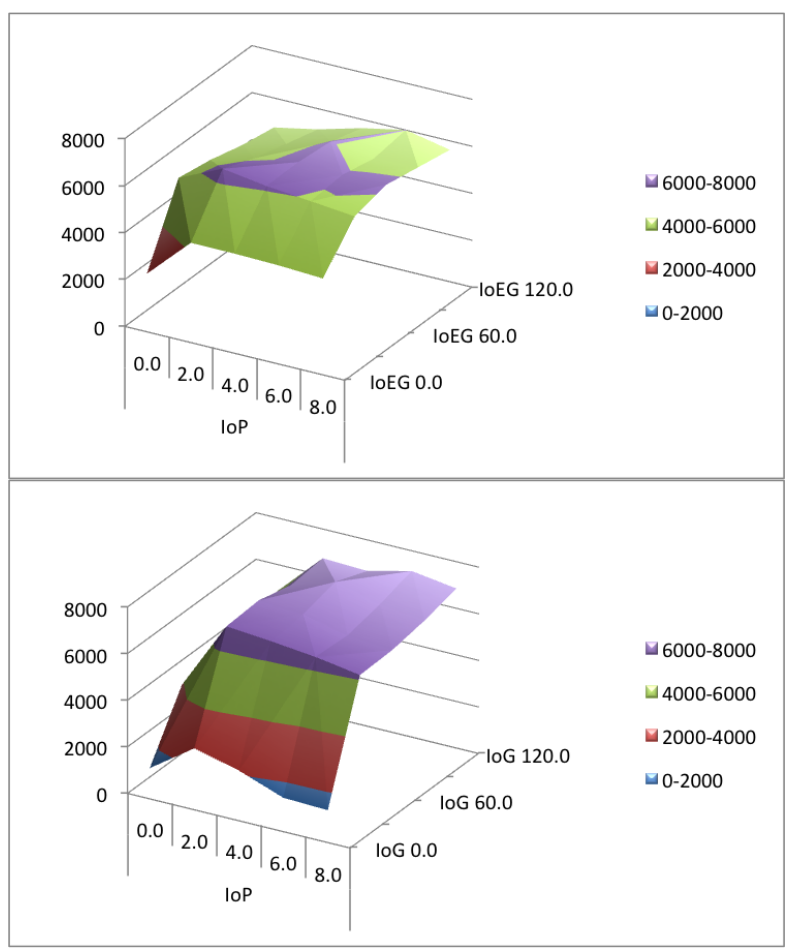

Fig. 6. The score of Ms. Pac-Man given the value of the Influence of Pills, and the Influences of edible Ghosts (top) and Ghosts (bottom) respectively.

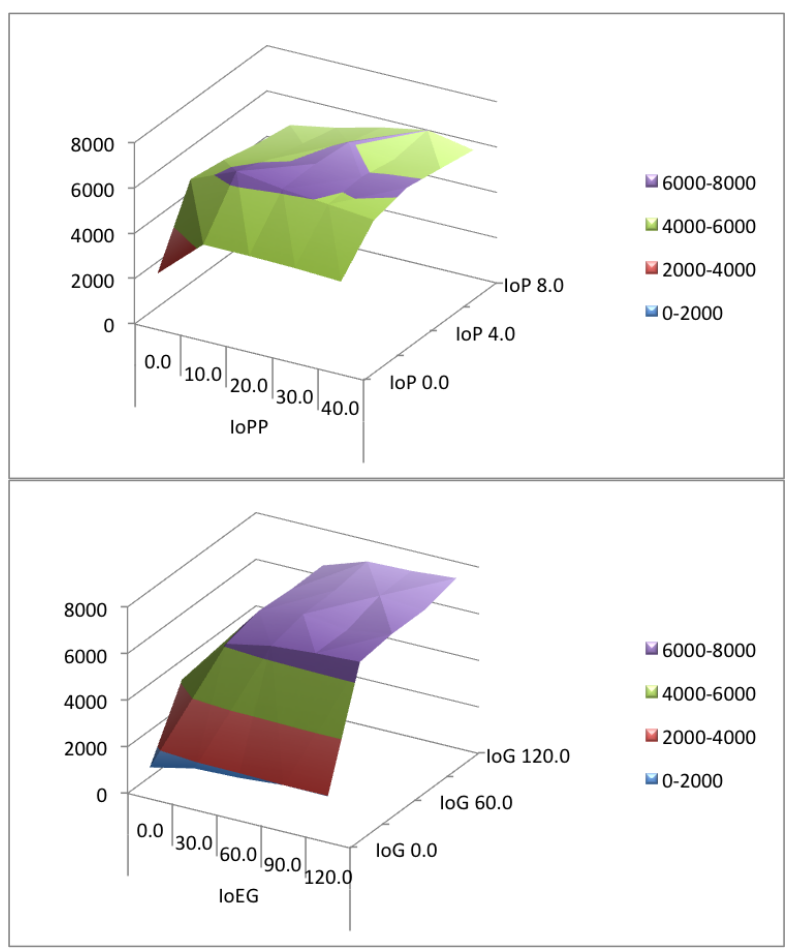

Fig. 7. The score of Ms. Pac-Man given the value of the Influences of Power Pills and Pills (top), and the Influences of edible Ghosts and Ghosts (bottom) respectively.

\section{ACKNOWLEDGMENTS}

several hundreds of steps of movements [11] or plan up to ten crossings ahead [12], our solution does only evaluate the options of one step actions.

Although this is a severe limitation of the ability to look into the future, sophisticated mechanisms such as trap escapes and ghost coordination may still be implemented. We would like to see how far we may stretch this kind of solution without increasing the look-ahead.

\section{CONClusions AND Future Work}

The influence map based controller seems to work reasonably well, especially in the middle of the map. Its shortcomings show in the corners, where the one step lookahead simply is not enough to manage to both be attracted by, and eat the pills in the corners, and at the same time avoid the repelling ghosts.

The results show a significant improvement compared to the previous studies using field-based approaches, peaking at 34170 points, with an average of 15775 using the optimal setup of parameters that we investigated.

Future work include improve the ghost coordination and to tune the influence maps further using some sort of learning algorithm.

We also consider the possible use of a weight map that influences the influences of moveable objects such as ghosts. We could then get Ms. Pac-Man to be more attracted to pills in long corridors when the ghosts are far away than if they are close by.
The authors would like to thank Blekinge Institute of Technology for supporting this work, Philipp Rolfshagen and Simon Lucas for providing the competition platform and the anonymous reviewers for their valuable comments.

\section{REFERENCES}

[1] S. Lucas, "Ms pac-man competition," ACM SIGEVOlution, vol. 2, no. 4, 2007.

[2] P. Rolfshagen and S. M. Lucas, "Ms pac-man versus ghost team cec 2011 competition," in Proceedings of IEEE Congress on Evolutionary Computation, 2011.

[3] J. Koza, Genetic Programming: On the Programming of Computers by Means of Natural Selection. MIT Press, 1992.

[4] M. Gallagher and A. Ryan, "Learning to play Pac-Man: An evolutionary, rule-based approach," in Proceedings of IEEE Congress on Evolutionary Computations, 2003.

[5] I. Szita and A. Lorincz, "Learning to play using low-complexity rulebased policies," Journal of Artificial Intelligence Research, vol. 30, no. 1, pp. 659-684, 2007.

[6] N. Wirth and M. Gallagher, "An influence map model for playing Ms. Pac-Man," in Proceedings of IEEE Computational Intelligence and Games (CIG), 2008.

[7] N. Bell, X. Fang, R. Hughes, G. Kendall, E. O'Reilly, and S. Qiu, "Ghost direction detection and other innovations for Ms. Pac-Man," in Proceedings of IEEE Computational Intelligence and Games (CIG), 2010.

[8] L. L. DeLooze and W. R. Viner, "Fuzzy Q-learning in a nondeterministic environment: Developing an intelligent Ms. Pac-Man agent," in Proceedings of IEEE Computational Intelligence and Games (CIG), 2009.

[9] E. Martin, M. Martinez, G. Recio, and Y. Saez, "Pac-mAnt: Optimization based on ant colonies applied to developing an agent for Ms. Pac-Man," in Proceedings of IEEE Computational Intelligence and Games $(C I G)$, 2010. 


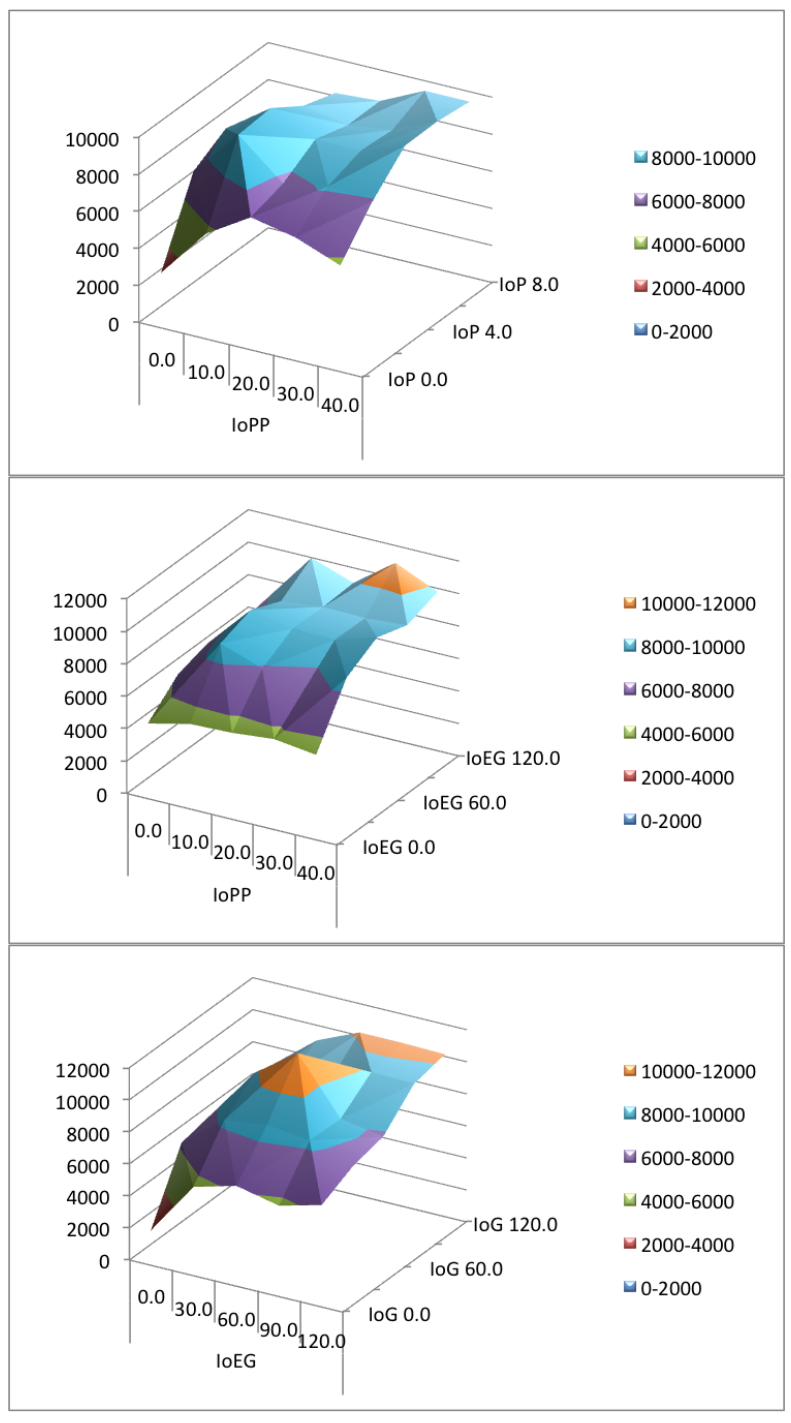

Fig. 8. The score of Ms. Pac-Man when $\mathrm{IoFoC}=90$, and $\mathrm{FoG}=-90$, given the value of the Influences of Power Pills and Pills (left), the Influences of Power Pills and edible Ghosts (right), and the influences of Pills and edible Ghosts (bottom) respectively.

[10] D. Robles and S. M. Lucas, "A simple tree search method for playing Ms. Pac-Man," in Proceedings of IEEE Computational Intelligence and Games (CIG), 2009.

[11] S. Samothrakis, D. Robles, and S. Lucas, "Fast approximate maxn monte carlo tree search for Ms Pac-Man," IEEE Transactions on Computational Intelligence and AI in Games, vol. 3, no. 2, 2011.

[12] R. Thawonmas and T. Ashida, "Evolution strategy for optimizing parameters in ms pac-man controller ice pambush 3," in Proceedings of IEEE Computational Intelligence and Games (CIG), 2010.

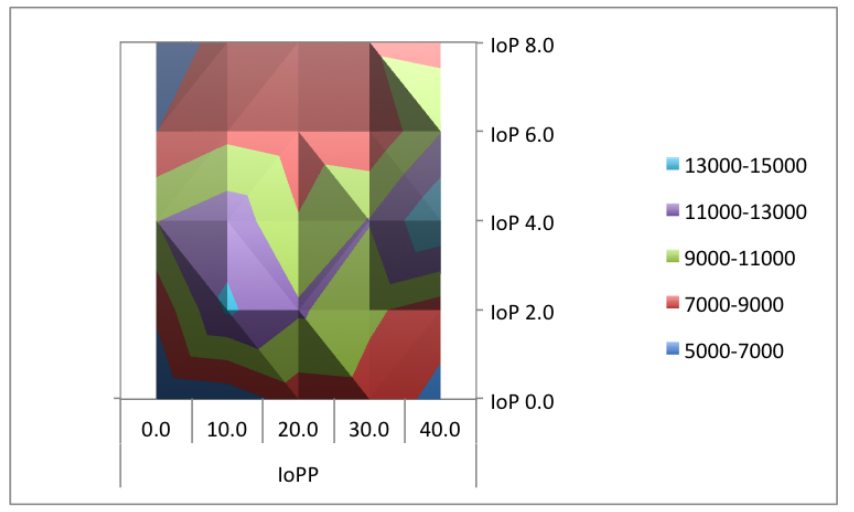

Fig. 9. The score of Ms. Pac-Man given the value of the Influences of Power Pills and Pills with $\mathrm{IoFoC}=90$, IoEG $=60$ and $\mathrm{IoG}=-90$.

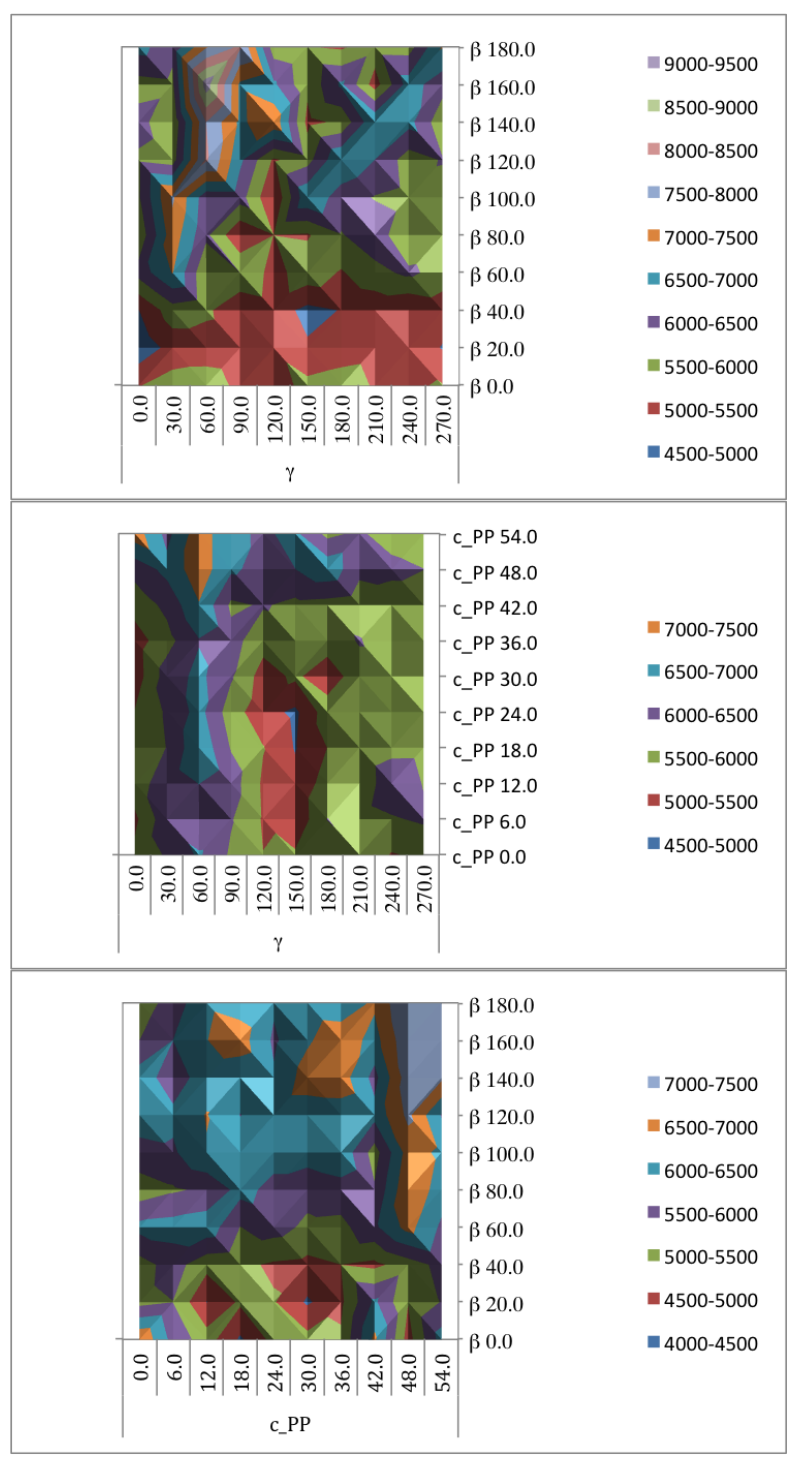

Fig. 10. The average score of Ms. Pac-Man when playing against our ghosts. The lesser the score, the better the ghosts manage. Just as in Figs. 3-8, the scores are averaged over the whole interval of the parameter not in the diagram. 